\title{
Simple analytical model for the magnetophoretic separation of superparamagnetic dispersions in a uniform magnetic gradient
}

\author{
J. S. Andreu, ${ }^{1,2, *}$ J. Camacho, ${ }^{2}$ J. Faraudo, ${ }^{1}$ M. Benelmekki, ${ }^{3}$ C. Rebollo, ${ }^{4}$ and L1. M. Martínez ${ }^{5}$ \\ ${ }^{1}$ Institut de Ciència de Materials de Barcelona (ICMAB-CSIC), Campus UAB, E-08193 Bellaterra, Spain \\ ${ }^{2}$ Departament de Física, Universitat Autònoma de Barcelona, Campus UAB, E-08193 Bellaterra, Spain \\ ${ }^{3}$ Centro de Fisica, Universidade do Minho, P-4710-057 Braga, Portugal \\ ${ }^{4}$ Sepmag Tecnologies, Parc Tecnològic del Valles, E-08290 Barcelona, Spain \\ ${ }^{5}$ Sepmag Technologies, 191 Peachtree St. NE, Atlanta Georgia 03030, USA
}

(Received 13 April 2011; revised manuscript received 27 June 2011; published 22 August 2011)

\begin{abstract}
Magnetophoresis - the motion of magnetic particles under applied magnetic gradient-is a process of great interest in novel applications of magnetic nanoparticles and colloids. In general, there are two main different types of magnetophoresis processes: cooperative magnetophoresis (a fast process enhanced by particle-particle interactions) and noncooperative magnetophoresis (driven by the motion of individual particles in magnetic fields). In the case of noncooperative magnetophoresis, we have obtained a simple analytical solution which allows the prediction of the magnetophoresis kinetics from particle characterization data (size and magnetization). Our comparison with new experimental results shows good quantitative agreement. In addition, we show the existence of a universal curve onto which all experimental results should collapse after proper rescaling. The range of applicability of the analytical solution is discussed in light of the predictions of a magnetic aggregation model [Soft Matter 7, 2336 (2011)].
\end{abstract}

DOI: 10.1103/PhysRevE.84.021402

PACS number(s): 82.70.Dd, 47.65.Cb, 85.70.Ay

\section{INTRODUCTION}

The manipulation of magnetic particles by the use of inhomogeneous magnetic fields (magnetophoresis) has emerged as a topic of great interest in a wide range of research and technological areas [1]. A broad spectrum of novel applications has been developed based on this concept: from environmental applications like wastewater treatments [2,3] and pollutant removal [4] to biomedical applications like protein isolation, drug delivery, magnetic hyperthermia for cancer treatment, and magnetic-particle imaging [5,6].

The particles employed in these applications are mainly superparamagnetic colloids with sizes ranging from a few nanometers to microns and with appropriate coatings, guaranteeing the stability and biocompatibility of the solutions. Superparamagnetic particles exhibit magnetizations of magnitudes similar to those of ferromagnetic materials, but they present no coercitivity nor remanence. This superparamagnetic behavior, which is of quantum origin, is limited to nanocrystals (NCs) of size below a critical size which depends on the material [7]. Larger superparamagnetic particles can be obtained as composite particles containing a nucleus of magnetic nanocrystals (typically iron oxide particles like magnetite $\mathrm{Fe}_{3} \mathrm{O}_{4}$ or its oxidized form maghemite $\gamma-\mathrm{Fe}_{2} \mathrm{O}_{3}$ ) inserted in a matrix of nonmagnetic material (such as polystyrene [8] or silica [9]). Since the superparamagnetic NCs contained inside the nucleus of the colloid are well separated, the behavior of the resulting colloid is also superparamagnetic. Thanks to this design, large magnetic moments can be obtained without losing the superparamagnetic response.

In this paper we study an issue that is common to some of the aforementioned applications of magnetic particles: the magnetic separation process. The idea behind magnetic

*jandreu@icmab.es separation is to take advantage of the distinctive magnetic response of the particles in solution to remove them from complex mixtures by the use of applied inhomogeneous magnetic fields [10]. In applications in closed containers $[11,12]$, the inhomogeneous magnetic field is used to drive the magnetic particles toward certain regions of the containing vessel enabling the removal of the "clean" liquid phase from the solid content. Differently, in continuous flow applications, magnetic particles are typically deflected from the direction of the laminar flow by a perpendicular magnetic field gradient, depending on their magnetic susceptibility, their size, and the flow rate. This approach is common to different field-flow fractionation or split-flow thin fractionation techniques [13] integrated in microfluidic devices enabling the trapping of magnetic particles or the fractionation of magnetic particles with different magnetic response [14].

In the different applications, magnetic particles are typically functionalized with proper chemical groups, which are designed to bind to specific nonmagnetic components, thus enabling the separation of nonmagnetic materials by combining the use of magnetic particles and magnetic fields. This combination has many advantages over traditional fixedbed separation methods, such as activated carbon adsorption for organics and affinity chromatography for proteins. In particular, the magnetic nanoparticles offer large exposed surface areas without the use of porous materials, which are often plagued by high mass-transfer resistances [15]. Therefore, it is not surprising that magnetic separation has been presented as an alternative to typical centrifugation and filtration steps in industrial processes as well as in laboratory applications.

Traditionally, the removal of magnetic particles (plus adsorbed biomaterial or pollutants) in solution is performed by the so-called high-gradient magnetic separation technique (HGMS) [11]. In an HGMS device, the dispersion containing 
the magnetic nanoparticles is pumped through a column filled with a packed bed of stainless steel fibers of the order of a few microns. These fibers are responsible for the high magnetic gradients inside the column once an external magnetic field is applied. This field can be generated in several different ways: by permanent magnets [3], electromagnets [15], or superconducting solenoids [16]. The HGMS technology, initially developed for magnetic clays, has been successfully employed [15] to capture functionalized nanoparticles with sizes larger than $\sim 10 \mathrm{~nm}$.

The most important drawback present in HGMS is the loss of control over the magnetic conditions under which the magnetic particles are removed. Basically, the external magnetic field applied induces highly inhomogeneous gradients in the separator. These inhomogeneous conditions common to the HGMS approach make it difficult to develop numerical and/or analytical solutions to the problem, which would help in a better understanding of the magnetophoretic mechanisms and enhancing its performance; for instance, by means of a better design of separators or a better choice of the magnetic particles used in specific applications. So far, theoretical work has basically focused on the modeling of individual dispersed magnetic nanoparticles in an HGMS column. Most of these works have been limited to simulate the behavior of nanoparticles around a single HGMS wire [11,17-19] or, more recently, monitoring the absorbance of superparamagnetic nanoparticles under the effects of a single permanent magnet [20,21]. Unfortunately, analytical solutions for (at least approximate) predictions of these magnetophoresis processes are not known.

In previous works [12,22-24] we have made use of a new concept of magnetic separation (so-called precision magnetophoresis) to effectively remove different types of superparamagnetic nanoparticles from solution. The key aspect here is that the separation process is based on the application of a homogeneous magnetic gradient to drive the removal of the particles, enhancing the control of the experimental conditions. Also, this simpler situation seems suitable for establishing a proper framework for the development of analytical models. Herein, we fill this gap by providing an analytical solution for the kinetics of the magnetophoresis separation process. The solution obtained is valid under certain restrictive but realistic conditions, which are explicitly discussed here. We also show the utility of the analytical model by comparing our predictions with experimental results obtained with superparamagnetic particles of different sizes and magnetizations. We expect that the availability of a simple analytical model will allow for a better understanding of the underlying physics of magnetic separation processes and also allow a rational design of applications.

\section{THEORY OF MAGNETOPHORESIS UNDER UNIFORM GRADIENT}

First of all, let us briefly describe the magnetic separation process that is under study. We consider here the case of a radial magnetic gradient, as in the experiments reported in Refs. $[12,23,24]$. In these experiments, an initially homogeneous dispersion of magnetic particles is placed inside a cylindrical cavity (of radius $L$ ) containing a uniform magnetic gradient pointing toward the walls of the vessel. Due to the magnetic gradient, particles move radially, reaching the vessel wall at the end of the process. At this final stage, the remaining liquid can be removed by pumping it from the center of the vessel, if desired, and the separation process will be completed (see video in [25] for a demonstration). Our objective in this section is to obtain an analytical expression for the kinetics of this magnetic separation process, relating separation times to basic properties of the particles (such as size and magnetization).

As explained in previous works [12,23], the experimental conditions consist of a dispersion of superparamagnetic particles (nanocrystals or composites) with radius $R$ and magnetization per unit mass $M(H)$ that are immersed in a fluid with viscosity $\eta$ at temperature $T$. Let us assume that, under these experimental conditions, the magnetophoretic separation is driven by the motion of the individual particles under the external magnetic field. In other words, we assume that particles do not form chains induced by the dipole-dipole interaction during the magnetic separation process (see, for example, Refs. [14,15,26]). We also note here that many superparamagnetic particles are designed for use in biomedical applications [5], which strictly require no chain formation even under strong fields [magnetic resonance imaging (MRI) contrast agents or hyperthermia treatments].

Neglecting the interaction between particles, we can obtain the magnetophoretic velocity of particles by noting that the viscous drag $F_{\text {vis }}=6 \pi \eta R v$ exerted by the solvent will be equal in magnitude (and opposite in direction) to the magnetic force over the particle $[10,14,15,26]$. Our approach follows the classical work of Senyei et al. [26] but, in our case, we have extended its validity to the nonlinear part of the magnetization response of the particle, contrary to what is typically required in other studies [14]. Under the effects of an external uniform magnetic gradient, the particle will experience a magnetic force $F_{\text {mag }}$ (in our particular case, in the radial direction, pointing toward the vessel wall) given by

$$
F_{\text {mag }}=m \mu_{0}\left(\frac{\partial H}{\partial r}\right)=\frac{4}{3} \pi R^{3} \rho_{p} M(H) \mu_{0}\left(\frac{\partial H}{\partial r}\right),
$$

where $\rho_{p}$ is the density of the particle, so its magnetic moment is given by $m(H)=(4 / 3) \pi R^{3} \rho_{p} M(H)$. Hence, in the stationary state the particles move with a velocity which depends both on the local values of the field $H$ and its gradient:

$$
v=\frac{2 R^{2}}{9 \eta} \mu_{0}\left(\frac{\partial H}{\partial r}\right) M(H) \rho_{p} .
$$

In order to proceed further we need to specify an analytical expression for the magnetic response $M=M(H)$ of the particles. For mathematical and conceptual simplicity, we assume that the magnetization curve $M=M(H)$ is described, to within a good approximation, by a Langevin function typical in theoretical descriptions of superparamagnetic systems [7,27-29]:

$$
M(H)=M_{\mathrm{s}} \mathcal{L}\left[b \mu_{0} H\right], \quad \mathcal{L}[x]=\operatorname{coth} x-\frac{1}{x},
$$

where $M_{\mathrm{s}}$ denotes the saturation magnetic moment per unit mass and $b^{-1}$ can be interpreted as a characteristic magnetic field required to reach saturation. For a superparamagnetic nanocrystal, $b$ can be computed if $M_{\mathrm{s}}$ and $R$ are known (see 
Ref. [28] and our supplemental material [30]). Now we can obtain the equation of motion for the particles and study the separation process. By combining equations (2) and (3) and noting that the magnetic field is linear and radial pointing to the vessel walls $[H=(\partial H / \partial r) r$ where $\partial H / \partial r$ is constant] we obtain the following result for the velocity of a particle located at a distance $r$ from the center of the system:

$$
v(r)=v_{\mathrm{s}} \mathcal{L}[\beta r / L],
$$

where $\mathcal{L}(x)$ is the Langevin function [see Eq. (3)] and we have defined

$$
\begin{gathered}
v_{\mathrm{s}}=\frac{2 R^{2}}{9 \eta} \mu_{0}\left(\frac{\partial H}{\partial r}\right) M_{\mathrm{s}} \rho_{p}, \\
\beta=b \mu_{0}\left(\frac{\partial H}{\partial r}\right) L .
\end{gathered}
$$

Physically, $v_{\mathrm{s}}$ is the magnetophoretic velocity of a particle with a magnetization equal to the saturation magnetization $M_{\mathrm{s}}$. The dimensionless parameter $\beta$ can be interpreted as the ratio between the magnetic field at the walls [the product $(\partial H / \partial r) L$ ] and the typical magnetic field $\left[b^{-1}\right.$, see Eq. (3)] required to bring a particle to magnetic saturation. Note that, according to Eq. (4), the particles reach their maximum magnetophoretic velocities $v_{\mathrm{s}}$ at positions $r$ verifying $\beta r / L \gg 1$. Now, we can obtain an exact, analytical expression for the separation time. Integrating Eq. (4) we obtain:

$$
\begin{gathered}
\frac{d r}{\mathcal{L}[\beta r / L]}=v_{\mathrm{s}} d t, \\
\int_{r_{0}}^{r} \frac{d r}{\operatorname{coth}(\beta r / L)-\frac{L}{\beta r}} \\
=\frac{L}{\beta}[\ln (\beta r / L \cosh (\beta r / L)-\sinh (\beta r / L))]_{r_{0}}^{r}=v_{\mathrm{s}} t,
\end{gathered}
$$

where $r_{0}$ is the radial coordinate of a particle at time $t=0$ and $r$ is its position at time $t$. Hence, the time $t$ needed by a particle to reach the wall of the vessel $(r=L)$ starting from an initial position $r_{0}>0$ [because the magnetic field at the center of the vessel is zero $H(0)=0$ ] is

$$
t=\frac{L}{\beta v_{\mathrm{s}}} \ln \left[\frac{\beta \cosh (\beta)-\sinh (\beta)}{\frac{\beta r_{0}}{L} \cosh \left(\beta r_{0} / L\right)-\sinh \left(\beta r_{0} / L\right)}\right] .
$$

Note that, if the particle is initially located at a position $r_{0}$ with a sufficiently high magnetic field, its magnetization is in the saturation regime $\left(\beta r_{0} / L \gg 1\right)$ and we have $\mathcal{L}[\beta r / L] \approx 1$ and $v \approx v_{\mathrm{s}}$, so that Eq. (8) reduces to $t=\left(L-r_{0}\right) / v_{\mathrm{s}}$.

Now, from Eq. (8) is straightforward to obtain the fraction of particles remaining in dispersion inside the system at a time $t$. For a given time $t$, all particles with initial radial positions smaller than the value $r_{0}$ given by Eq. (8) will be still in solution (not at the wall). Hence, at that specific time $t$, the number of particles $N(t)$ remaining inside the separator is equal to the number of particles with initial radial coordinate smaller than $r_{0}$, which is proportional to $\pi r_{0}^{2}$. Therefore, the fraction $N(t) / N(0)$ of particles inside the magnetic separator is given by

$$
f \equiv \frac{N(t)}{N(0)}=\left(\frac{r_{0}}{L}\right)^{2}
$$

Using Eqs. (8) and (9) we obtain the separation time $t$ at which a given fraction of particles $f$ is still in dispersion (not at the walls):

$$
t=\frac{L}{\beta v_{\mathrm{s}}} \ln \left[\frac{\beta \cosh (\beta)-\sinh (\beta)}{\beta \sqrt{f} \cosh (\beta \sqrt{f})-\sinh (\beta \sqrt{f})}\right] .
$$

In other words, Eq. (10) gives the time $t$ needed to reach the state with a fraction of particles $f$ still in solution. Note that, in the limiting case of $\beta \gg 1$ and $\beta \sqrt{f} \gg 1$, Eq. (10) reduces to

$$
f \approx\left(1-\frac{v_{\mathrm{s}}}{L} t\right)^{2} .
$$

In practice, $\beta$ is typically between 10 and 100 (see next section), so Eq. (11) can usually be employed to estimate the initial kinetics of the separation process. Physically, Eq. (11) describes the separation process assuming that the magnetization of particles is at saturation, whereas Eq. (10) takes into account the full $M(H)$ dependence.

At this point, it is important to explicitly discuss under which conditions this requirement of no chain formation under a magnetic field is fulfilled. The classical criterion against chain formation induced by the dipole-dipole interaction derived in the context of ferrofluids (see, for example, p. 37 in Ref. [28]) requires that the magnetic coupling constant, defined as

$$
\Gamma=\frac{\mu_{0} m_{\mathrm{s}}^{2}}{2 \pi d^{3} k_{B} T},
$$

verifies $\Gamma \ll 1$. In Eq. (12) $m_{\mathrm{s}}$ is the magnetic moment of a particle at saturation and $d$ is its diameter. Physically, $\Gamma$ is the ratio between the magnetic energy associated to the dipoledipole attraction (which tends to induce the formation of chains of particles in the dipole direction) and the thermal energy (which tends to disaggregate). However, our recent simulation results show that this classical criterion is not fulfilled in dispersions of superparamagnetic particles. For example, we have shown that, for $\Gamma=3$ and a volume fraction $\phi_{0}=$ $5.23 \times 10^{-4}$, no aggregation is found (see Fig. 2 Ref. [31]), in disagreement with the classical criterion. In general, our simulations have shown that the aggregation behavior depends both on $\Gamma$ and the volume fraction $\phi_{0}$ (defined as the volume fraction of particles uniformly dispersed in solution before applying the magnetic field). Combining simulation results with a thermodynamical model [31] we have shown that the aggregation behavior of superparamagnetic dispersions is controlled by the dimensionless parameter $N^{*}$ given by

$$
N^{*}=\sqrt{\phi_{0} e^{\Gamma-1}} .
$$

As shown in Ref. [31], in order to observe aggregation, the condition $N^{*}>1$ should be verified. In this case, the number density of chains of length $s$ follows

$$
n_{s} \propto e^{-s / N^{*}} \text {. }
$$

and the average length of chains is thus $\bar{n} \approx N^{*}$. For more details and a comparison with simulations, see Ref. [31]. Our results also agree with recent small angle neutron scattering results [32]. Typical values of $\Gamma$ and $N^{*}$ of superparamagnetic colloids employed in the laboratory will be discussed in detail 
in the next section. Here it is enough to remark that all analytical results derived in this section are valid under the condition $N^{*}<1$.

Another issue to be considered in applying our theoretical model is the possible effect of sedimentation due to gravity. In principle, sedimentation will be irrelevant in the separation process if the vertical distance sedimented during magnetophoresis is smaller than the vertical size of the system. In our experimental situations, we will have always a sedimentation velocity much smaller than magnetophoresis velocity; in addition, the radius is much smaller than the height of the container.

\section{USE OF ANALYTICAL MODEL IN ANALYSIS OF MAGNETOPHORESIS EXPERIMENTS}

\section{A. Comparison of predictions with experiments}

Now we will explore the use of the analytical results obtained in the previous section to understand the kinetics of actual magnetophoresis separation experiments. In particular, we will show the usefulness of Eq. (10) in predicting magnetophoresis kinetics in the case $N^{*}<1$. We will also discuss the limitations of our analytical model by briefly discussing the case $N^{*}>1$, for which no analytical solution is yet available.

For the sake of concreteness, we will consider results for dispersions of 4 different samples of superparamagnetic particles (see Table I), differing in size and composition but all of them representative of the many different kinds of particles employed in biotechnological applications. Further technical details (synthesis, characterization) are given in the supplemental material or in the indicated references. In all cases, our magnetophoretic separation experiments were performed using precision magnetophoresis systems from SEPMAG [33], the SEPMAG LAB $1 \times 25 \mathrm{ml} 2042$ and 2042 plus (in both cases, $L=1.5 \mathrm{~cm}$ ). The magnetophoresis kinetics is monitored by measuring the opacity of the sample, as in previous works [12,23,24] (we also describe again the full experimental methodology in the accompanying supplemental material). Here it suffices to say that the measured opacity (after appropriate normalization) provides a good estimate of the fraction $f$ of particles remaining in solution.

Let us first consider sample $\mathrm{S} 1$, which consists of maghemite $\gamma-\mathrm{Fe}_{2} \mathrm{O}_{3}$ nanocrystals about $12 \mathrm{~nm}$ in size dispersed in water at a $10 \mathrm{~g} / \mathrm{L}$ concentration. It is known that the colloidal stability (in the absence of a magnetic field) of these small nanocrystals in dispersions could be an issue. In our case, we ensure the stability by electrostatic stabilization.

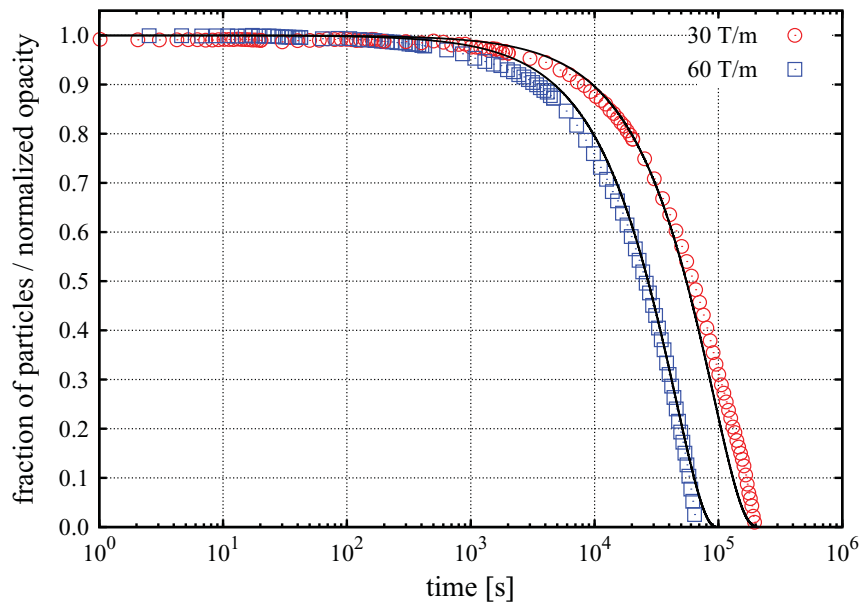

FIG. 1. (Color online) Kinetics of magnetophoretic separation of a $10 \mathrm{~g} / \mathrm{L}$ dispersion of superparamagnetic $\gamma-\mathrm{Fe}_{2} \mathrm{O}_{3}$ nanocrystals (sample S1). Solid lines are the predictions of Eq. (10) (with no free parameters) and symbols are the experimental results under $30 \mathrm{~T} / \mathrm{m}$ (circles) and $60 \mathrm{~T} / \mathrm{m}$ (squares).

For full details of sample preparation, stabilization, and characterization, see the supplemental material. As shown in Table I, $N^{*}<1$, so Eq. (10) applies in this case. The values of the required quantities $v_{\mathrm{s}}$ and $\beta$ were computed by using the data in Table I in Eqs. (5) and (6). For example, under a $60 \mathrm{~T} / \mathrm{m}$ gradient we obtain $v_{\mathrm{s}}=1.59 \times 10^{-7} \mathrm{~m} / \mathrm{s}$ and $\beta=61.7$. In Fig. 1 we show the theoretical predictions and the experimental results for two different magnetic gradients (30 and $60 \mathrm{~T} / \mathrm{m}$ ). Our results (see Fig. 1) show that Eq. (10) can be successfully applied to predict the magnetophoretic behavior from averaged properties of the sample, such as particle radius $R$ and saturation dipole $m_{\mathrm{s}}$. Of course, in real samples there are always factors difficult to control (such as a certain degree of polydispersity differing from batch to batch of identically produced particles) which may cause deviations from the ideal behavior predicted by Eq. (10). Also, the measurement of the full magnetophoresis curve is particularly challenging since, in some cases, it requires more than one day to complete and additional spurious effects (such as changes of viscosity of water due to temperature variations during the experiment) may reduce the agreement between theory and experiment. In spite of these limitations, the agreement between theory and experiment is rather satisfactory for this sample. For example, at a field gradient of $60 \mathrm{~T} / \mathrm{m}$ we predict that half of the dispersion $(f=0.5)$ is removed at separation times of $7.5 \mathrm{~h}$, close to the experimental value of $7 \mathrm{~h}$.

TABLE I. Physical parameters for the different particles employed in the magnetophoretic experiments shown in Figs. 1 and $2: 2 R$ is the diameter, $\rho_{p}$ is the density, $m_{\mathrm{s}}$ is the magnetic dipole at saturation, and the quantities $b, \Gamma$, and $N^{*}$ are defined in Eqs. (1), (12), and (13), respectively.

\begin{tabular}{|c|c|c|c|c|c|c|}
\hline Sample & $2 R[\mathrm{~nm}]$ & $\rho_{p}\left[\mathrm{~g} / \mathrm{cm}^{3}\right]$ & $m_{\mathrm{s}}[\mathrm{J} / \mathrm{T}]$ & $b\left[\mathrm{~T}^{-1}\right]$ & $\Gamma$ & $N^{*}$ \\
\hline $\mathrm{S} 1\left(\gamma-\mathrm{Fe}_{2} \mathrm{O}_{3}\right)$ & 12 & 4.86 & $3.0 \times 10^{-19}$ & 68 & 2.5 & 0.1 \\
\hline S2 Core $\left(\gamma-\mathrm{Fe}_{2} \mathrm{O}_{3}\right) /$ Shell $\left(\mathrm{SiO}_{2}\right)$ & 82 & 2.40 & $3.0 \times 10^{-18}$ & 9.3 & 0.8 & 0.02 \\
\hline S3 Core $\left(\gamma-\mathrm{Fe}_{2} \mathrm{O}_{3}\right) /$ Shell $\left(\mathrm{SiO}_{2}\right)$ & 157 & 2.35 & $1.7 \times 10^{-17}$ & 13.8 & 3.5 & 0.2 \\
\hline Estapor ${ }^{\circledR}$ M1-020/50 & 200 & 1.10 & $2.5 \times 10^{-16}$ & $\mathrm{~N} / \mathrm{A}$ & $\sim 10^{3}$ & $\sim 10^{21}$ \\
\hline
\end{tabular}




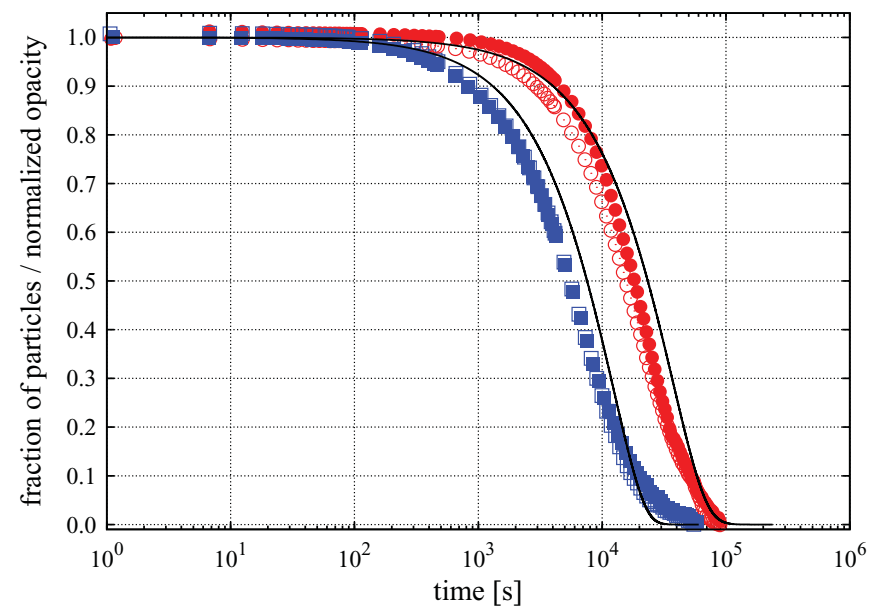

FIG. 2. (Color online) Comparison of magnetophoretic separation kinetics of colloidal dispersions containing different composite superparamagnetic particles obtained in experiments (symbols) with the predictions of Eq. (10) (solid line) without adjustable parameters. Circles correspond to sample S2 and squares to sample S3 with a magnetic gradient of $60 \mathrm{~T} / \mathrm{m}$ (open and filled symbols correspond to different repetitions of the same experiment).

Now let us consider the results for a different kind of superparamagnetic particles. Samples S2 and S3 correspond to a dispersion in water of core shell composite particles described in Ref. [9] (also denoted by samples S2 and S3 in that reference) which were designed as image contrast agents in MRI. In these composite nanoparticles, the shell is made of microporous silica and the core contains several $6.5 \mathrm{~nm}$ $\gamma-\mathrm{Fe}_{2} \mathrm{O}_{3}$ nanocrystals previously synthesized in Ref. [34]. The average size of the composite particles is $82 \mathrm{~nm}$ in the case of $\mathrm{S} 2$ and $157 \mathrm{~nm}$ in the case of S3, as determined by transmission electron microscopy (TEM) (although there is some polydispersity in size, as discussed in Ref. [9]). In this case, the dispersions had a concentration of $1 \mathrm{~g} / \mathrm{L}$ and $N^{*}<1$ so Eq. (10) is expected to apply. It should be noted that, in applications, these particles need to be employed always under conditions with $N^{*}<1$ to avoid magnetic aggregation and allow their use as image contrast agents.

In Fig. 2 we show the results under a gradient of $60 \mathrm{~T} / \mathrm{m}$. The values for the saturation velocity $v_{\mathrm{s}}$ and dimensionless parameter $\beta$, computed by using the data in Table I in Eqs. (5) and (6), are $v_{\mathrm{s}}=2.19 \times 10^{-7} \mathrm{~m} / \mathrm{s}$ and $\beta=9.5$ for $\mathrm{S} 2$ and $v_{\mathrm{s}}=6.46 \times 10^{-7} \mathrm{~m} / \mathrm{s}$ and $\beta=14.1$ for S3. Again, Eq. (10) can be applied to predict, with reasonable approximation, the magnetophoretic behavior from average particle data. For example, predicted times for $f=0.5$ are 6 hours (sample S2) and 2 hours (sample $\mathrm{S} 3$ ), which are reasonably close to the experimental values of 4.3 hours and 1.5 hours, respectively.

We note here that the agreement between theory and experiments for S2 and S3 is less satisfactory than that observed for S1 (compare Fig. 1 with Fig. 2). This could be attributed to the fact that the fit of the magnetic response of samples S2 and S3 to the Langevin function Eq. (3) is less accurate than in the case of sample S1 (see the supplemental material for details on the magnetic characterization of the samples). We expect that the agreement could be further improved by using more sophisticated functions to model the $M(H)$ response (such as those proposed in Ref. [29]). However, this better fit of the $M(H)$ response will make it impossible to obtain an analytical solution for $f$. Here we prefer to maintain the model as simple as possible, even at the cost of losing some accuracy in the results (which is in fact not essential in practice).

Up to now, we have discussed the case of magnetophoresis due to single-particle motion $\left(N^{*}<1\right)$, which is a situation in which our analytical model holds. It is reasonable to expect that, in the case $N^{*}>1$ (formation of chains of magnetic particles induced by the external field), the observed magnetophoresis kinetics will be faster than expected from our analytical calculations. However, it is not obvious how large the difference between the single-particle kinetics described by our model and cooperative kinetics will be. To this end, we also include a comparison with experimental results, obtained in our previous study [12], with commercial Estapor ${ }^{(\mathrm{R})}$ M1-020/50 particles, which correspond to extremely large $N^{*}$ (see Table I). These commercially available particles are made of magnetic nanocrystals embedded in a polystyrene matrix and are employed in immunoassay applications (in these applications, reversible magnetic aggregation under applied field is often a desired effect). An example of the experimental results obtained in Ref. [12] are shown in Fig. 3. These results correspond to a solution of $1 \mathrm{~g} / \mathrm{L}$ concentration under a $30 \mathrm{~T} / \mathrm{m}$ gradient. The kinetics of single-particle magnetophoresis was estimated using $v_{\mathrm{s}} \simeq 4 \times 10^{-6} \mathrm{~m} / \mathrm{s}$ (as calculated from the data in Table I). Since we do not pretend in this case to provide an accurate prediction, we employ, for simplicity, Eq. (11) for $f$, which assumes that individual particles always move with their maximum possible velocity $v_{\mathrm{s}}$, which corresponds to the saturation magnetization. It is clear that the kinetics observed for Estapor ${ }^{(\mathrm{R})}$ M1-020/50 particles is orders of magnitude faster than the kinetics expected from the $v_{\mathrm{s}}$ value for these particles. In fact, the magnetophoretic process is very fast and complete separation is obtained in less than 2 minutes.

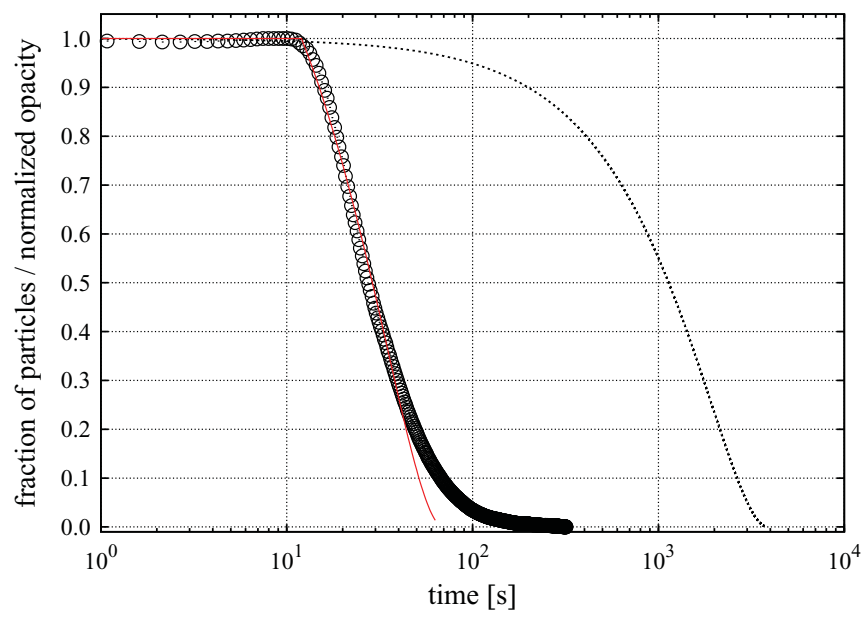

FIG. 3. (Color online) Comparison between cooperative magnetophoresis observed with commercial Estapor ${ }^{(\mathrm{R})} \mathrm{M} 1-020 / 50$ particles (symbols) and the single-particle magnetophoresis kinetics $\left(N^{*}<1\right)$ ideally expected by these particles moving at their maximum velocity $v_{\mathrm{s}}$, according to Eq. (11) (dashed line). The solid line is a fit of the initial decay to Eq. (11) leaving the magnetophoretic velocity as a fitting parameter, which gives $v_{\mathrm{s}}^{\mathrm{agg}} \approx 2.6 \times 10^{-4} \mathrm{~m} / \mathrm{s}$. 
Hence, the comparison shown in Fig. 3 is consistent with the existence of a cooperative process in the experiments, as we proposed originally in Ref. [12]. In that reference, the observed fast kinetics was attributed to the formation of elongated aggregates. In fact, the results of Fig. 3 can be employed to obtain a crude estimate of the velocity and size of these aggregates. The velocity of the aggregates can be estimated as follows: We interpret the experimental data in Fig. 3 as corresponding to an initial regime (for times up to $12 \mathrm{~s}$ ) in which aggregates are formed and $f \approx 1$ followed by magnetophoresis kinetics obeying Eq. (11) with a certain velocity for the aggregates $v_{\mathrm{s}}^{\mathrm{agg}}$. Fitting the experimental data for $t$ up to $100 \mathrm{~s}$ (see fit in Fig. 3), we obtain $v_{\mathrm{s}}^{\text {agg }} \approx 2.6 \times 10^{-4}$ $\mathrm{m} / \mathrm{s}$; that is, we estimate that aggregates move 65 times faster than individual particles. If we approximate the aggregates as ellipsoids of semiaxis $a R$ and $c R(a \geqslant c)$, it is easy to show that the magnetophoretic velocity of the ellipsoid at magnetic saturation is given by $v_{\mathrm{s}}^{\mathrm{agg}}=\left[a c^{2} / C_{D}(a, c)\right] v_{\mathrm{s}}$, where $v_{\mathrm{s}}$ is the velocity of a single particle [as given by Eq. (5)] and $C_{D}(a, c)$ is the drag coefficient given by [35] $\left(\delta^{2} \equiv a^{2}-c^{2}\right)$

$$
C_{D}=\frac{8}{3}\left[\frac{1}{\delta} \ln \left(\frac{a+\delta}{a-\delta}\right)-\frac{2 a^{2}}{\delta^{3}}\left(\frac{\delta}{a}-\operatorname{arcsinh}(\delta / c)\right)\right]^{-1} .
$$

Hence, our previous estimate of $v_{\mathrm{s}}^{\mathrm{agg}} \approx 65 v_{\mathrm{s}}$ is consistent with ellipsoidal aggregates of different dimensions. We provide just two examples: ellipsoids with semiaxes $a \approx 11.5, c \approx 4.7$ and $a \approx 32, c \approx 4.6$. The first one would contain around 250 particles and the second one about 700 particles. This indicates that aggregates in the cooperative magnetophoresis process may contain several hundred particles, a situation quite different from the one studied here (and the subject of future work).

We can conclude this subsection by saying that the results presented here support the view proposed in the previous section that two very different magnetophoresis processes are possible under a constant magnetic gradient. Single particle magnetophoresis (described by our analytical model) is observed for $N^{*}<1$ whereas cooperative magnetophoresis (involving the formation of large elongated aggregates of particles induced by the magnetic field) is observed for $N^{*} \gg 1$.

\section{B. Possibility of a universal curve}

Interestingly, our theoretical results imply that all experimental magnetophoretic results obtained for $N^{*}<1$ should collapse onto a unique, universal curve after properly rescaling quantities. In fact, by defining a dimensionless time $\tau \equiv t v_{\mathrm{s}} / L$ and function $J(x) \equiv \ln (x \cosh (x)-\sinh (x))$, expression (10) becomes

$$
\tau=\frac{1}{\beta}\left[J(\beta)-J\left(\beta f^{1 / 2}\right)\right] .
$$

Therefore, one obtains that $\tau$ is a function of $f$ and is dependent on the specific magnetophoretic system through the dimensionless parameter $\beta$ defined in Eq. (6). One can invert this function to get the fraction of particles in solution $f$ in terms of the dimensionless time $\tau$ and parameter $\beta$. A
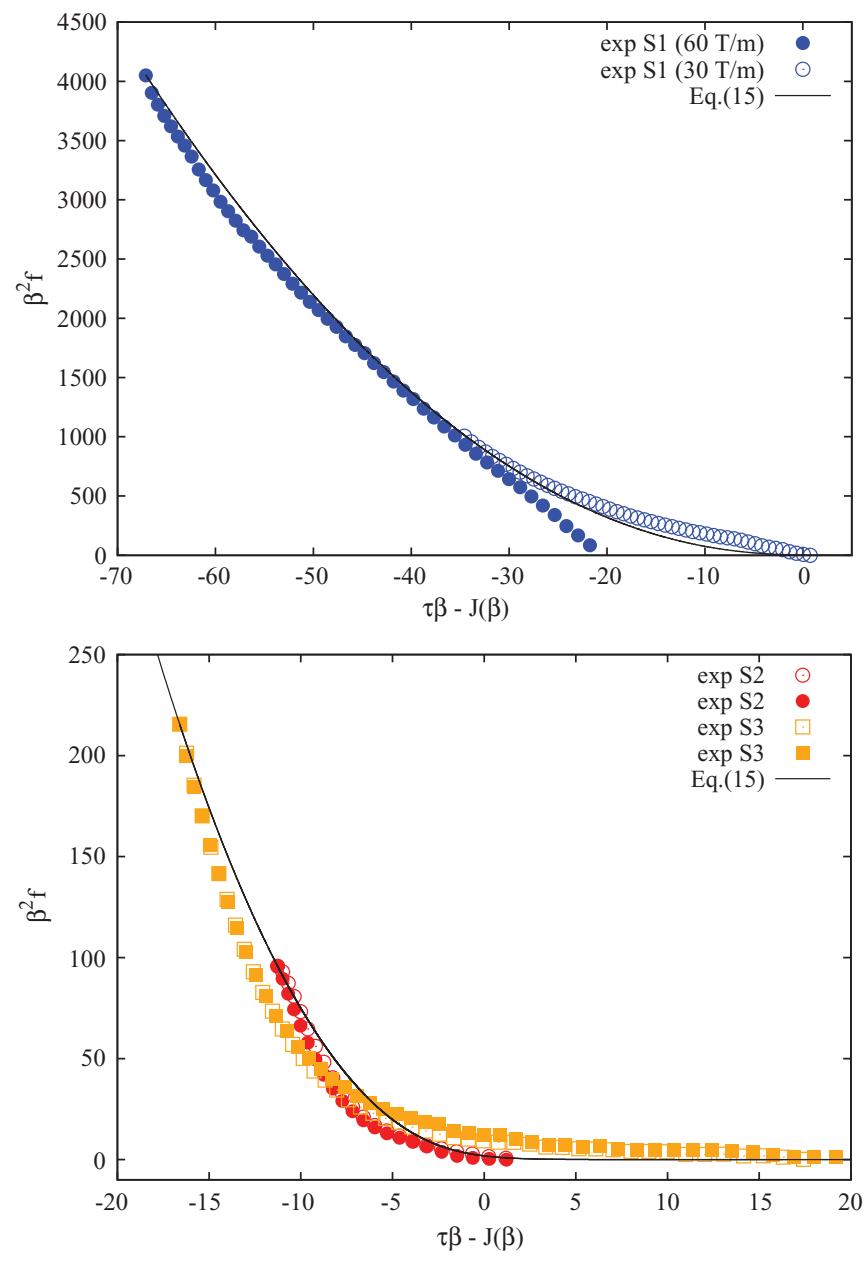

FIG. 4. (Color online) Comparison of theoretical predictions for a universal kinetic curve [Eq. (17), solid line] with experimental results (symbols). Top panel shows a comparison using experimental data for $\gamma-\mathrm{Fe}_{2} \mathrm{O}_{3}$ nanocrystals (sample $\mathrm{S} 1$ ) under $30 \mathrm{~T} / \mathrm{m}$ (open symbols) and $60 \mathrm{~T} / \mathrm{m}$ (filled symbols). Bottom panel shows a comparison using experimental results for composite particles. Circles correspond to sample S2 and squares to sample S3 (open and filled symbols correspond to different repetitions of the same experiment).

direct manipulation of Eq. (16) yields

$$
\beta^{2} f=g(J(\beta)-\beta \tau),
$$

where $g(x) \equiv\left[J^{-1}(x)\right]^{2}$ and $J^{-1}(x)$ is the inverse of function $J(x)$. Equation (17) shows that the rescaled fraction of particles $\beta^{2} f$ is a universal function $g$ of a rescaled time defined as $J(\beta)-\beta \tau$.

In Fig. 4 we show a plot of this universal curve compared with the experimental results for samples $\mathrm{S} 1$ (under two different gradients of 30 and $60 \mathrm{~T} / \mathrm{m}$ ) and samples S2 and S3 (under $60 \mathrm{~T} / \mathrm{m}$ ). These systems correspond to very different values for the scaled quantities, so we prefer to show the results in two different panels for easier visibility. The results for sample S1 (Fig. 4, top panel) show, in general, good agreement with the theoretical predictions, although deviations are found at small opacities, where measurements have less accuracy. For samples of composite particles (S2 and S3, Fig. 4, bottom panel), the agreement is satisfactory. 


\section{CONCLUSIONS}

In this paper, we have analyzed magnetophoretic separation of superparamagnetic particles of different types under wellcontrolled magnetophoretic conditions (in a system based on a radial, uniform magnetic gradient). Depending on the properties of the particles and the dispersion, we have proposed two different kinetic regimes for magnetophoretic separation, characterized by a dimensionless quantity denoted as $N^{*}$ [see Eq. (13)]. For $N^{*}<1$, the kinetics of the magnetophoresis process is dominated by the motion of single particles in a magnetic gradient (noncooperative magnetophoresis) and, consequently, the time scales for magnetic separation are large (many hours in the experiments performed here). In this case, it is possible to obtain an analytical solution for the kinetics of the magnetophoresis process with no free parameters and which shows a satisfactory agreement with experimental results. Our analytical results also show the existence of a universal curve [see Eq. (17) and Fig. 4], onto which all experimental results should collapse after proper scaling (provided that they correspond to the noncooperative regime, $N^{*}<1$ ).

In the case of large $N^{*}$ (which corresponds to particles with high magnetic dipole and moderate or large concentrations), the magnetophoresis process is enhanced by the formation of long chains of particles which move rapidly in the magnetic gradient. This cooperative magnetophoresis process (described in our previous work [12]) is characterized by a kinetics orders of magnitude faster than that expected from the single-particle model. No analytical models are available for this case yet, so this important case will be the subject of further work.
It is also interesting to note that we have focused here on the prediction of the magnetophoresis behavior of particles with known magnetic properties [i.e., a well-characterized $M(H)$ curve]. However, in some practical applications it could be of interest to obtain an estimation of the $M(H)$ curve as a byproduct of a magnetophoresis process by fitting the obtained kinetics with our analytical results: Eqs. (5), (6), and (10) (provided that $N^{*}<1$ ).

Finally, as a limitation of our model, we note that polydispersity effects are not taken into account. Although synthesis methods are advancing rapidly and provide very monodisperse particles, some polydispersity is still possible. However, it is difficult to include this effect rigorously in an analytical model. An important difficulty is the lack of analytical knowledge of the distributions of sizes and magnetizations in real samples. In any case, our comparisons with experimental results shows that the assumption of a mean size for the particles is a good first-order approximation.

\section{ACKNOWLEDGMENTS}

This work is supported by the Spanish Government Grants No. FIS2009-13370-C02-02, No. PET2008-02-8101/02, No. INNPACTO IPT-010000-2010-6, and No. CONSOLIDER-NANOSELECT-CSD2007-00041. J.F. and J.C. are also supported by the Catalan Government Grant No. 2009SGR164. We thank Anna Roig and Elena Taboada (ICMAB-CSIC) for kindly supplying us samples S2 and S3 and their characterization data, as well as for extensive discussions.
[1] C. T. Yavuz, A. Prakash, J. T. Mayo, and V. L. Colvin, Chem. Eng. Sci. 64, 2510 (2009).

[2] C. De Latour, IEEE Trans. Magn. 9, 314 (1973).

[3] G. Mariani, M. Fabbri, F. Negrini, and P. L. Ribani, Sep. Purif. Technol. 72, 147 (2010).

[4] C. T. Yavuz, Science 314, 964 (2006).

[5] J. L. Corchero and A. Villaverde, Trends Biotechnol. 27, 468 (2009).

[6] K. M. Krishnan, IEEE Trans. Magn. 46, 2523 (2010).

[7] C. P. Bean and J. D. Livingstone, J. Appl. Phys. 30, 120S (1959).

[8] D. Leun and A. K. Sengupta, Environ. Sci. Technol. 34, 3276 (2000).

[9] E. Taboada, R. Solanas, E. Rodríguez, R. Weissleder, and A. Roig, Adv. Funct. Mater. 19, 2319 (2009).

[10] G. Friedman and B. Yellen, Curr. Opin. Colloid Interface Sci. 10, 158 (2005).

[11] R. Gerber, M. Takayasu, and F. J. Friedlaender, IEEE Trans. Magn. 19, 2115 (1983).

[12] G. de las Cuevas, J. Faraudo, and J. Camacho, J. Phys. Chem. C 112, 945 (2008).

[13] N. Pamme and A. Manz, Anal. Chem. 76, 7250 (2004).

[14] H. Watarai, M. Suwa, and Y. Iiguni, Anal. Bioanal. Chem. 378, 1693 (2004).

[15] G. D. Moeser, K. A. Roach, W. H. Green, T. A. Hatton, and P. E. Laibinis, AIChE J. 50, 2835 (2004).
[16] S. K. Baik, D. W. Ha, R. K. Ko, and J. M. Kwon, Physica C 470, 1831 (2010).

[17] M. Takayasu, R. Gerber, and F. J. Friedlaender, IEEE Trans. Magn. 19, 2112 (1983).

[18] L. P. Davies and R. Gerber, IEEE Trans. Magn. 26, 1867 (1990).

[19] T. Y. Ying, S. Yiacoumi, and C. Tsouris, Chem. Eng. Sci. 55, 1101 (2000).

[20] V. Schaller et al., J. Appl. Phys. 104, 093918 (2008).

[21] L. E. Helseth and T. Skodvin, Meas. Sci. Technol. 20, 095202 (2009).

[22] J. Faraudo and J. Camacho, Colloid Polym. Sci. 288, 207 (2010)

[23] M. Benelmekki, C. Caparros, A. Montras, R. Goncalves, S. Lanceros-Mendez, and Ll. M. Martínez, J. Nanopart. Res. 13, 3199 (2010).

[24] M. Benelmekki, A. Montras, A. J. Martins, P. J. G. Coutinho, and Ll. M. Martínez, J. Magn. Magn. Mater. 323, 1945 (2011).

[25] See video at [http://www.youtube.com/watch?v=BVipEdKoMh8].

[26] A. Senyei, K. Widder, and G. Czerlinski, J. Appl. Phys. 49, 3578 (1978).

[27] R. Kaiser and G. Miskolczy, J. Apply. Phys. 41, 1064 (1970).

[28] R. E Rosensweig, Ferrohydrodynamics, 1st ed. (Cambridge University Press, New York, 1985). 
[29] D. X. Chen, A. Sanchez, E. Taboada, A. Roig, N. Sun, and H. C. Gu, J. Appl. Phys. 105, 083924 (2009).

[30] See Supplemental Material at http://link.aps.org/supplemental/ 10.1103/PhysRevE.84.021402 for details on the synthesis of sample $\mathrm{S} 1$, the magnetic characterization of all samples, and a description of the magnetophoretic measurements.

[31] J. S. Andreu, J. Camacho, and J. Faraudo, Soft Matter 7, 2336 (2011).
[32] M. Barrett, A. Deschner, J. P. Embs, and M. C. Rheinstädter, Soft Matter 7, 6678 (2011).

[33] SEPMAG Tecnologies [http://www.sepmag.eu].

[34] E. Taboada, E. Rodríguez, A. Roig, J. Oró, A. Roch, and R. N. Muller, Langmuir 23, 4583 (2007).

[35] H. Lamb, Hydrodynamics, 6th ed. (Cambridge University Press, Cambridge, 1993). 